\title{
Preventing Plasmon Coupling Between Gold Nanorods Improves the Sensitivity of Photoacoustic Detection of Labelled Stem Cells In Vivo
}

Joan Comenge ${ }^{+*}$, Oihane Fragueiro ${ }^{\ddagger}$, Jack Sharkey ${ }^{\S}$, Arthur Taylor ${ }^{\S}$, Marie Held ${ }^{\dagger}$, Neal C. Burton", Brian Kevin Park ${ }^{\S}$, Bettina Wilm ${ }^{\S}$, Patricia Murray ${ }^{\S}$, Mathias Brust ${ }^{\ddagger}$, and Raphaël Lévy $^{\dagger *}$

† Institute of Integrative Biology, University of Liverpool, Liverpool L69 7ZB, United Kingdom

‡ Department of Chemistry, University of Liverpool, Liverpool L69 7ZD, United Kingdom

$\S$ Institute of Translational Medicine, University of Liverpool, Liverpool L69 3BX, United Kingdom

"iThera Medical GmbH, Zielstattstrasse 13, Munich, Germany

* Address correspondence to: J.Comenge@liverpool.ac.uk, rapha@liverpool.ac.uk

\section{Supporting Information}

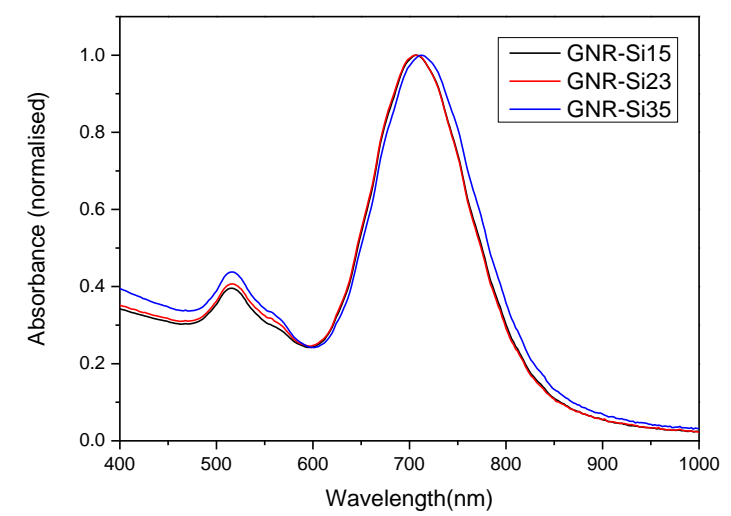

Figure S1. Vis-NIR spectra of GNRs incubated with DMEM. Optical spectra of GNRs did not change after 24 hours incubation in cell culture medium (DMEM + 10\% FBS+ $1 \%$ L-Glu, without phenol red) indicating absence of any aggregation. DMEM without GNRs was used as blank. 


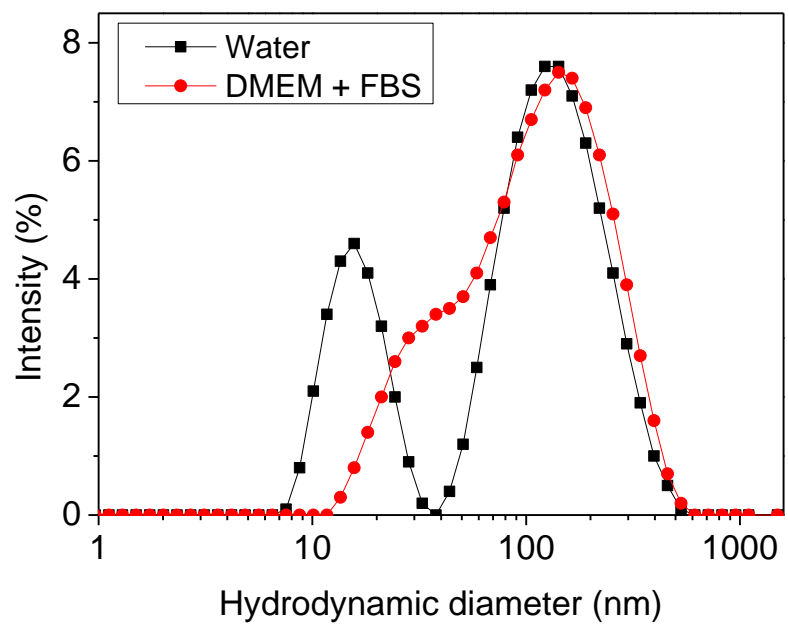

Figure S2. Hydrodynamic diameter as measured by DLS of GNRs-Si23 in water and in cell culture medium (DMEM + 10 \% FBS). The absence of aggregation was confirmed. A slight increase in diameter was observed due to absorption of proteins from the media. Note also that anisotropy of GNRs is translated in 2 peaks in the DLS measurements due to the rotational motion of non-spherical particles. ${ }^{1}$ The same behaviour was observed for GNR-Si15 and GNR-Si35.
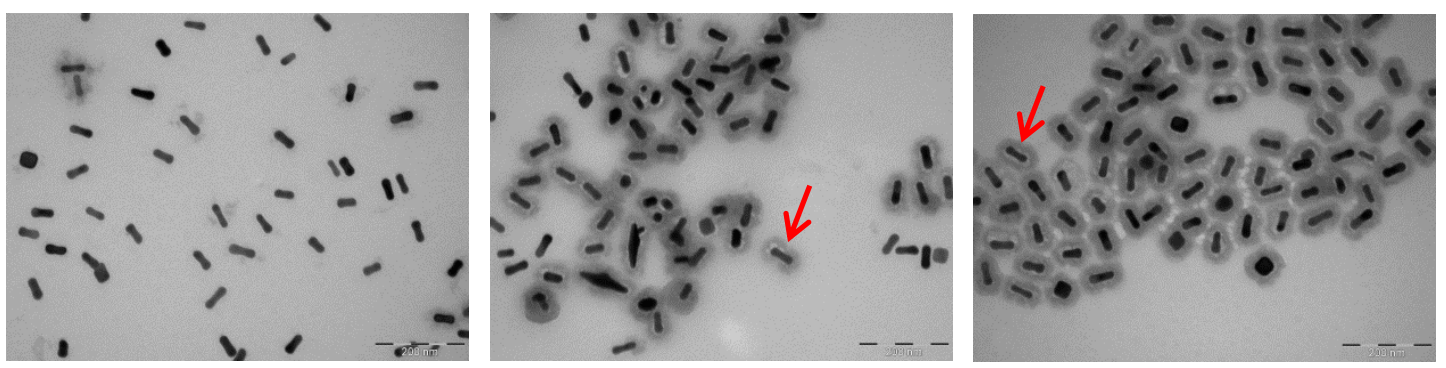

Figure S3. Silica shell degradation in DMEM. TEM analysis of GNR-Si15 (a), GNR-Si23 (b), GNR-Si35 (c) after 12 hour incubation in tissue culture media. The silica shell was almost totally degraded in the GNR-Si15 whilst it was much better preserved for the thicker shells. A closer look at the GNRs revealed that the etching started at the metal interface as previously reported ${ }^{2}$ (indicated with red arrows).

\section{Photothermal imaging}

Photothermal imaging is an optical technique in which energy from a time-modulated laser beam (heating beam) is absorbed by the GNRs present in the focal volume and converted to thermal energy. The local change of temperature around a GNR induces a spatially modulated refractive index profile in its vicinity, within which a second, non-resonant probe laser beam is scattered to produce a distinctive frequency-shifted scattered field. GNRs that are much smaller than the optical detection limit can thus be visualised at high resolution and sensitivity. Compared to TEM imaging of single cell slices, photothermal imaging of a $70 \times 70 \mu \mathrm{m}$ field of view therefore provided us with an 
overview over the loading of several whole cells with GNRs without requiring any additional labelling or sample processing.

All images were acquired using a custom photothermal microscope ${ }^{3}$ built around the body of a Nikon Eclipse Ti-U inverted microscope. Prior to photothermal imaging, the samples were allowed to equilibrate fully to room temperature for at least an hour. The photothermal excitation (heating) laser (523 nm, $0.25 \mathrm{~mW}$; frequency-doubled Nd:YAG, Ventus Laser Quantum, Germany) was modulated at a frequency of $459.5 \mathrm{kHz}$ using an accousto-optical modulator (Isomet Corporation, UK). The excitation beam was overlaid with a non-resonant probe laser $(633 \mathrm{~nm}, 2.5 \mathrm{~mW}$; JDS Uniphase Corporation, USA) via a cold mirror (ThorLabs, USA). The superimposed beams were focused onto the sample via a Zeiss Plan Achromat 63× oil immersion objective (numerical aperture: 1.4). The sample was placed on a piezo scanning stage (MCL502385, MadCity Labs, USA), which allows the movement of the sample in three dimensions over the fixed laser spot. Pixel-by-pixel scanning was facilitated by a piezoelectric stage driver (MCL NanoDrive 85, MadCity Labs, USA) controlled through a Nanonis RC4 module and Nanonis program (SPECS Zurich, Switzerland). The transmitted and forward scattered light was collected via a Zeiss Achroplan 40x water immersion objective (numerical aperture: 0.8 ) and passed through a red-pass filter (ThorLabs, USA) to block the excitation laser. The red component was focused on a photodiode of the balanced photo receiver (Model $210710 \mathrm{MHz}$ adjustable photo receiver, New Focus, USA). A lock-in amplifier (DSP 7260, Signal Recovery, USA) was used to identify the scattered component of the probe beam that corresponds to the modulation frequency (i.e. $459.5 \mathrm{kHz}$ ). A Nanonis SC4 Acquisition Module (SPECS Zurich, Switzerland) was used for signal acquisition. The signal was averaged and a grayscale pixel value (voltage) was generated. The pixel-by-pixel values were then converted into a photothermal image and saved in the Nanonis-native .sxm format. Gwyddion (http://gwyddion.net) ${ }^{4}$ was used to display the photothermal images, using the built-in Warpp-mono colour gradient. 


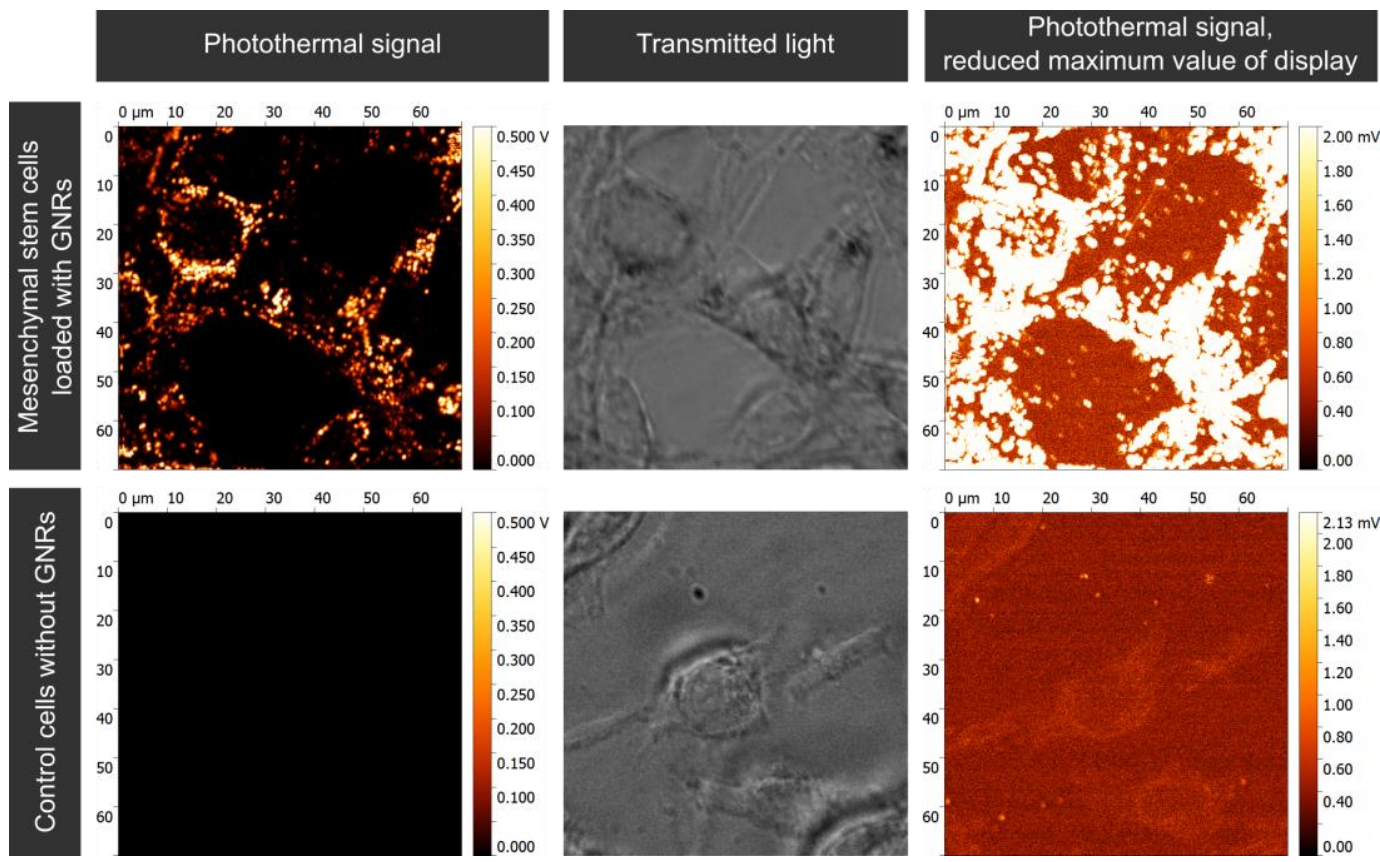

Figure S4. Photothermal evaluation of GNR uptake. Images of cells treated with 30 pM GNRs-Si35 for $24 \mathrm{~h}$ and control cells that were not exposed to GNRs. The top left image shows several GNR-loaded cells in the same field of view. GNRs are located in puncta in the perinuclear regions as well as cell extensions. The two bottom rows show control cells that were not labelled with GNRs. In the first column, no signal can be seen. Upon reducing the maximum displayed voltage value by a factor of 235 (third column), an endogenous mitochondrial photothermal signal becomes visible.

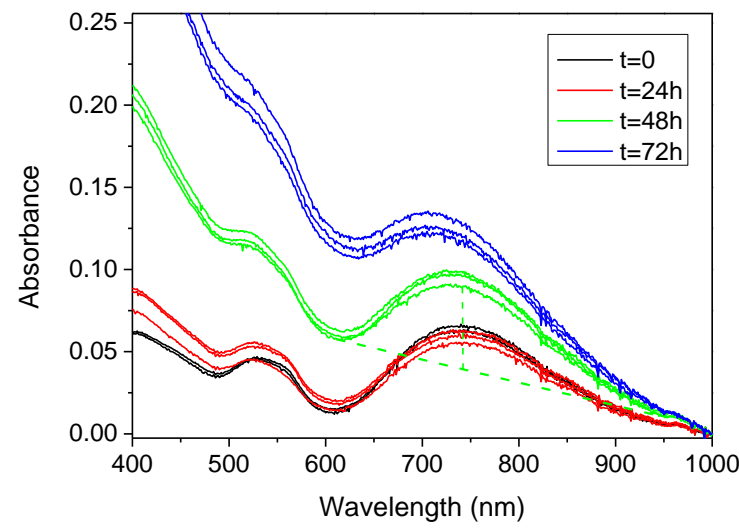

\begin{tabular}{ll}
\hline Time (h) & Absorbance peak \pm SD \\
\hline $\mathbf{0}$ & $0.053 \pm 0.003$ \\
$\mathbf{2 4}$ & $0.047 \pm 0.001$ \\
$\mathbf{4 8}$ & $0.054 \pm 0.003$ \\
$\mathbf{7 2}$ & $0.045 \pm 0.001$ \\
\hline
\end{tabular}

Figure S5. Vis-NIR analysis of plasmon bands for GNR-Si35 in MSC-D1 cells after various lengths of culture. MSC-D1 were incubated 24h with GNR-Si35 as explained in the main text. Then, cells were washed and incubated with fresh media. Cells from 3 wells were immediately harvested and Vis-NIR spectra were taken $(t=0)$. The rest of the cells were maintained in culture. The harvesting and Vis-NIR analysis were repeated after 24,48 , and 72 hours. It is observed that GNRs were stable in all conditions since there was no broadening of the plasmon band. The number of cells increased with time, as can be seen by higher scattering at short wavelengths (all conditions have been off-lined at $1000 \mathrm{~nm}$ to facilitate the visualisation). However, the absorbance of the peak did not change dramatically even at longer times, which suggests that exocytosis of GNRs (if any) was limited to a very small fraction of the material. The absorbance of the peak was calculated as illustrated for the first $48 \mathrm{~h}$ time point. This enables the cell scattering contribution to the extinction spectra to be discounted. 

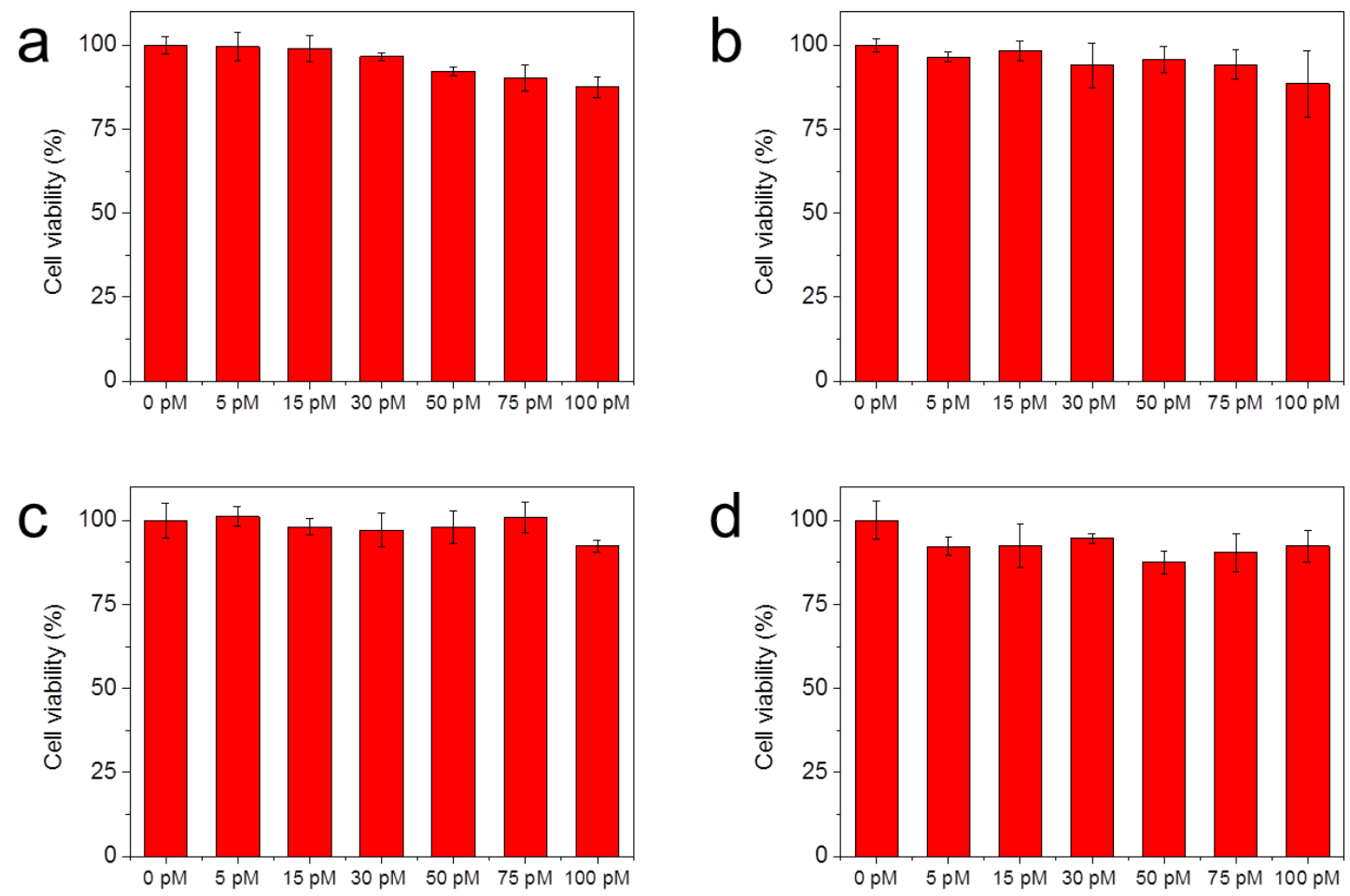

Figure S6. Cell viability from $\mathbf{0}$ to $\mathbf{7 2}$ hours after labelling. MSC-D1 viability immediately after $24 \mathrm{~h}$ incubation with GNRs at different concentrations (a) and $24 \mathrm{~h}(\mathrm{~b}), 48 \mathrm{~h}$ (c), and $72 \mathrm{~h}$ (d) after labelling. 

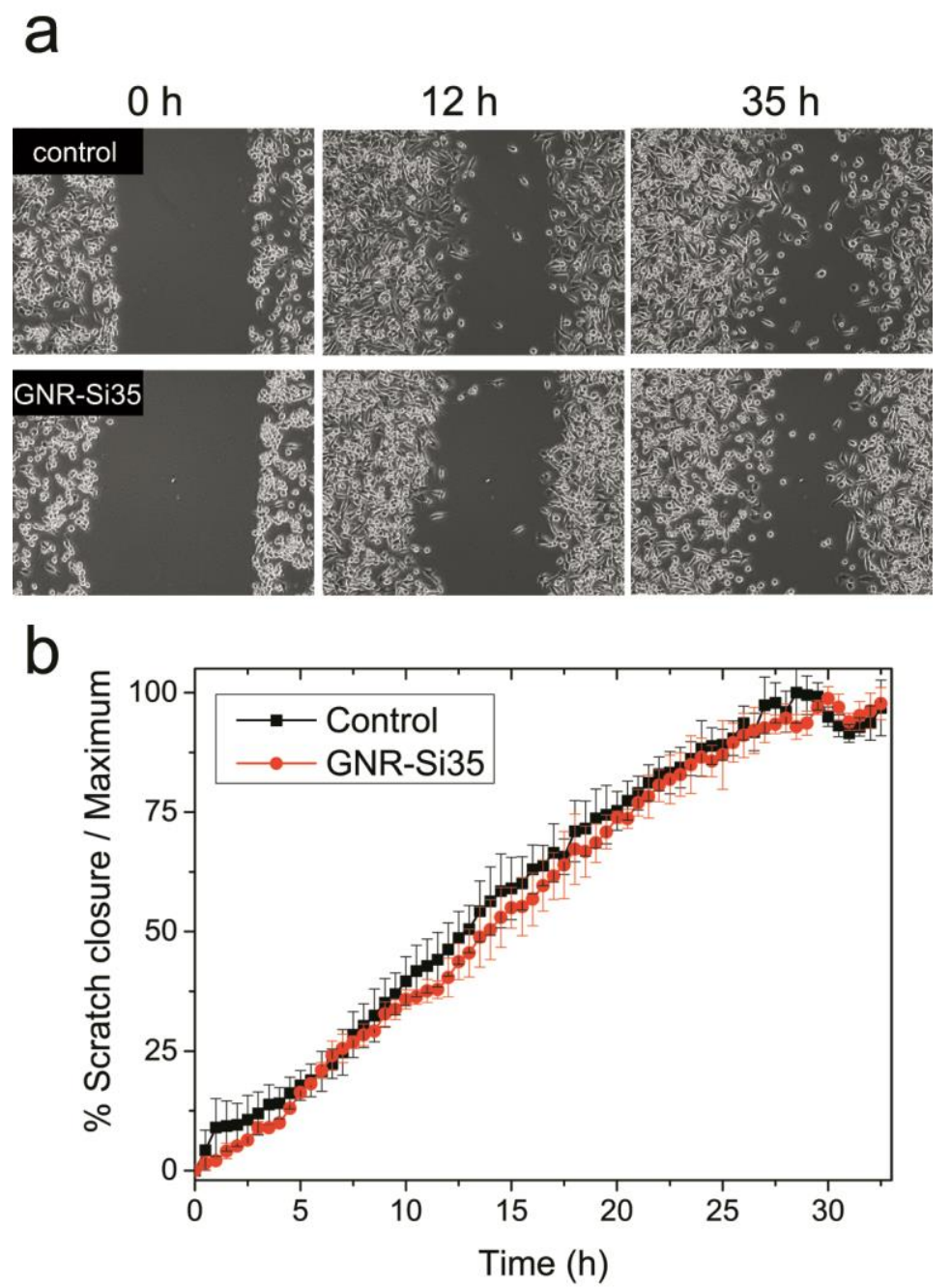

Figure 57. Migration assay. Cells were labelled as explained in the main article and the closure of a scratch was monitored for $35 \mathrm{~h}$. Representative pictures of control cells and GNR-Si35-labelled cells after 0,18 , and $35 \mathrm{~h}$ are shown in (a). The \% of scratch closure respect to the maximum closure is shown in (b). 4 different regions for each condition were analysed. 


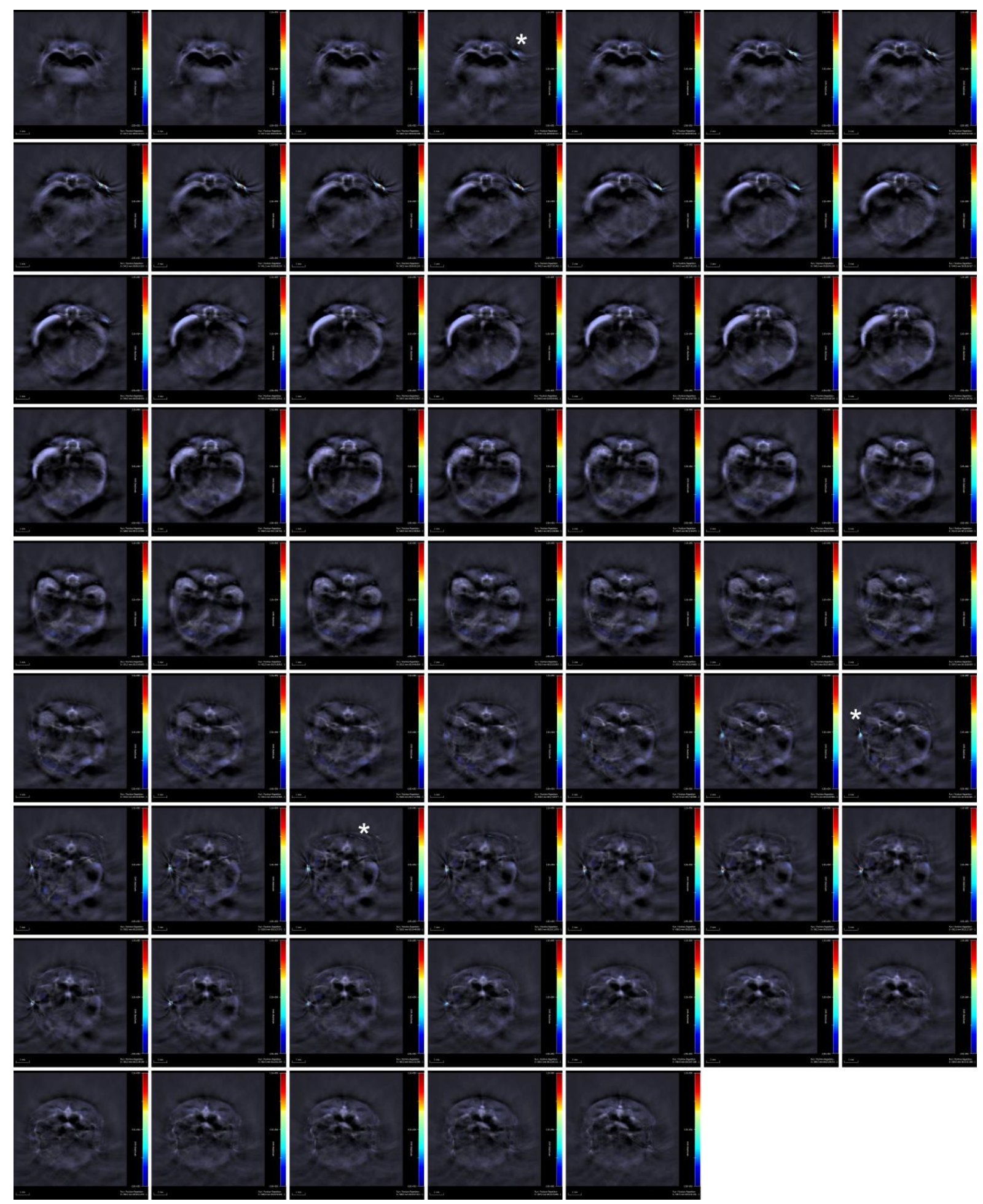

Figure S8. Whole animal scanning. Multispectral processed images of all positions at day 1 . It can be observed that MSOT intensity was only observed in places where cells were injected (indicated with *). No MSOT signal was observed anywhere else. Colour bar range is $-2 \times 10^{3}$ to $1.1 \times 10^{5} \mathrm{MSOT}$ a.u. 

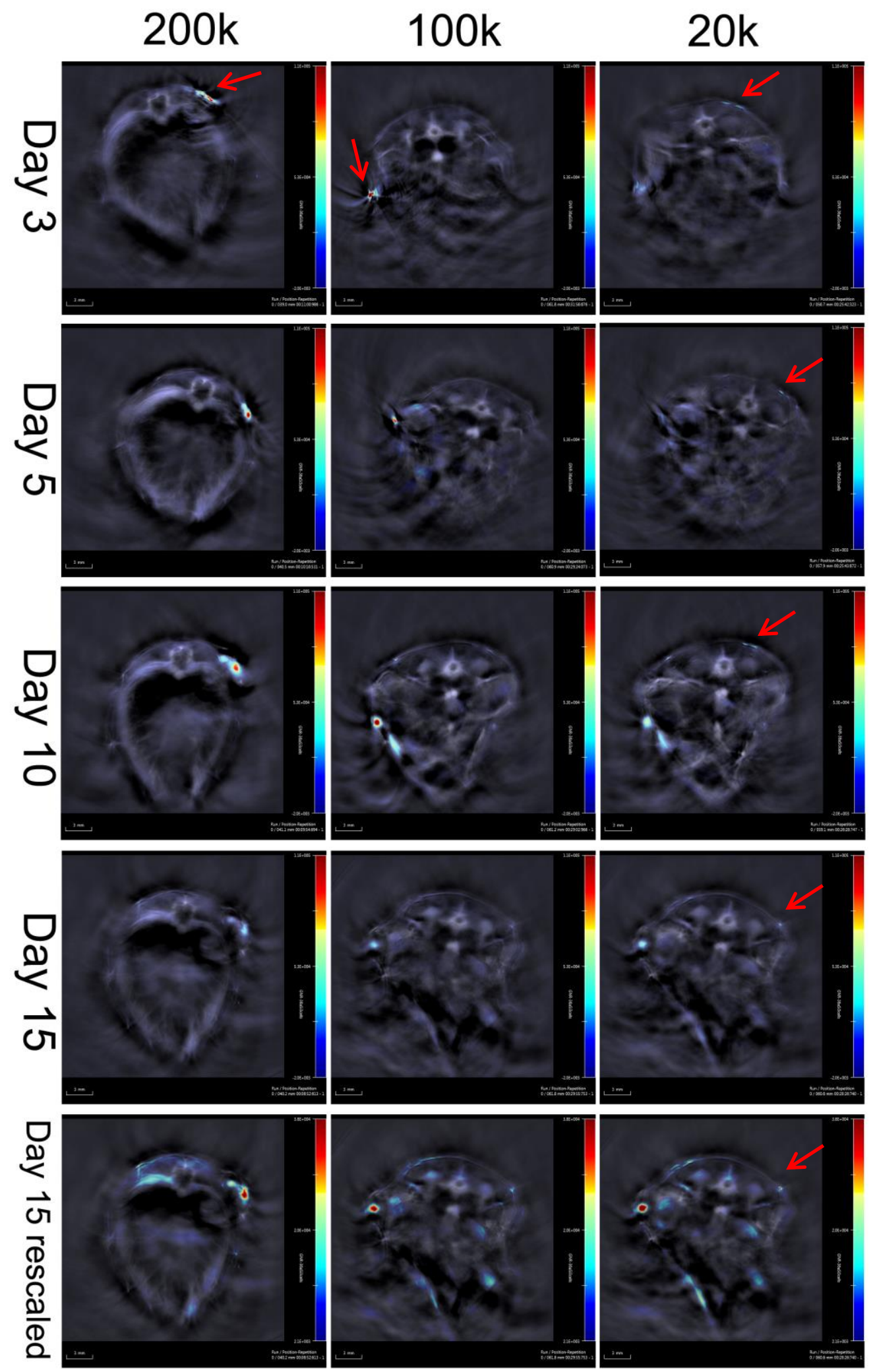

Figure 59. Representative images of the 3 cell clusters at the different time points. Colour scale represents MSOT arbitrary units (a.u.) after multispectral processing. It goes from $-2 \times 10^{3}$ to $1.1 \times 10^{5}$ a.u. in all cases. Day 15 is also shown with an additional colour scale (day 15 rescaled) to show that although cell clusters did lose intensity, they could still be identified. In this latter case the scale goes from $2.1 \times 10^{3}$ to $3.8 \times 10^{4}$ a.u., this is why regions of background showed up in this new range. 


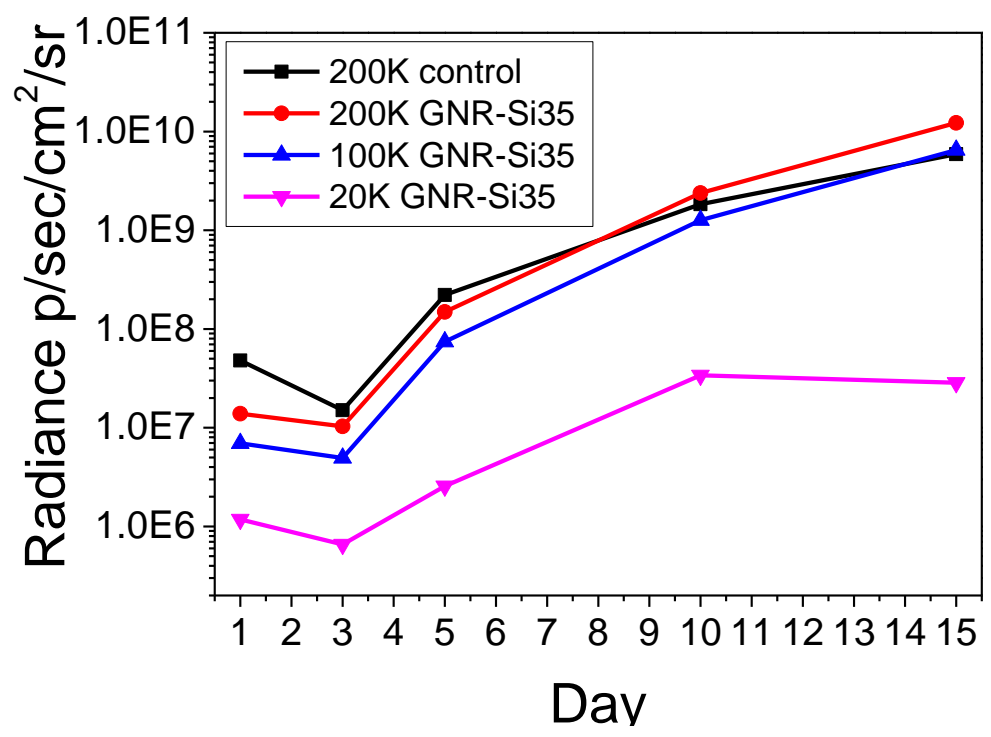

Figure S10. Bioluminescence quantification of the different cell clusters. The general trend is an arrested growth (or decrease) during the first five days (lag phase) followed by an exponential growth. This trend was also observed when analysing cell cluster volumes by MSOT imaging (see main text Figure 11). 

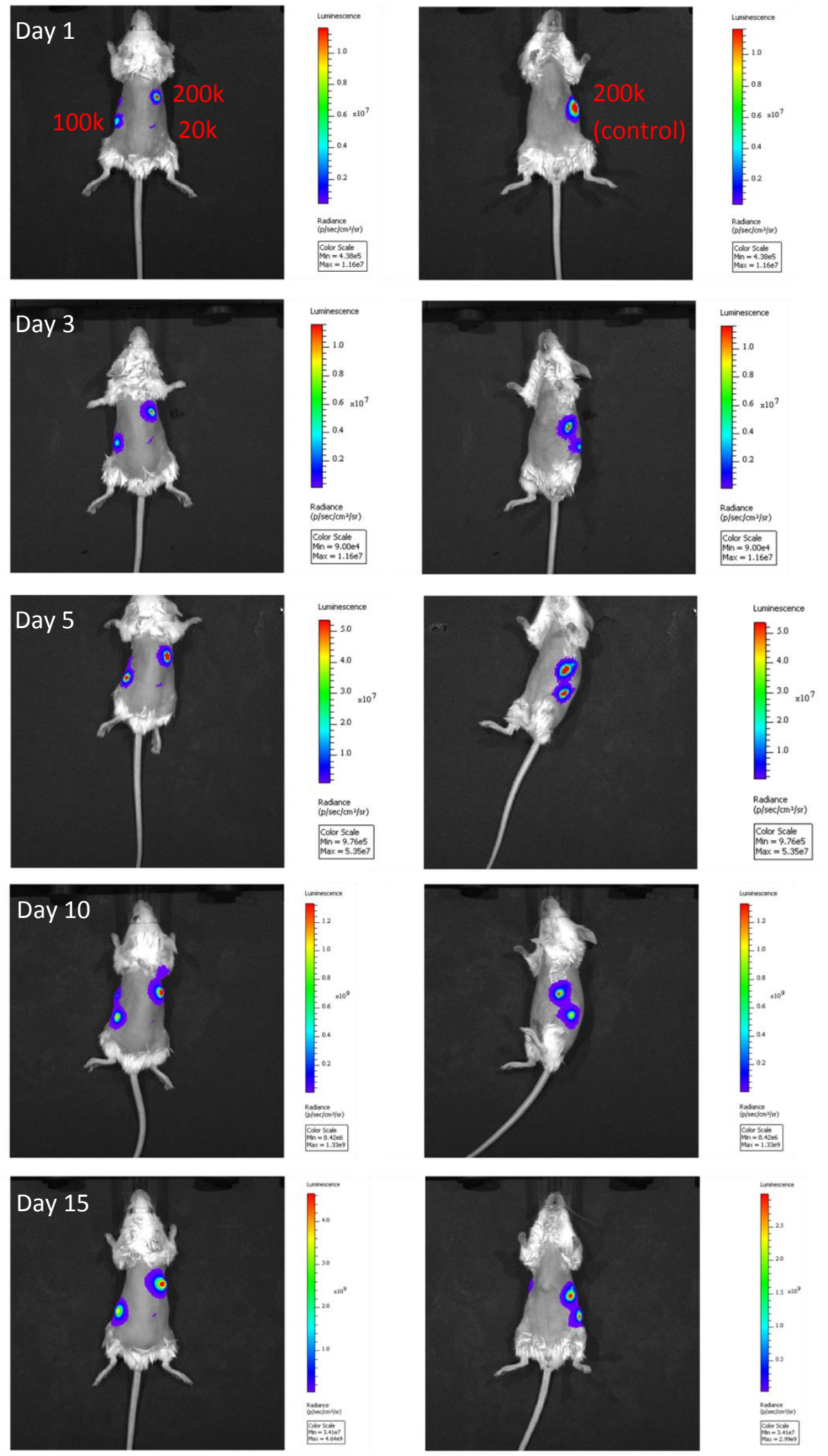

Figure S11. Bioluminescence imaging of the cell clusters at different days. Labelled cells can be observed in the left column. The same mouse had to be imaged in ventral position to observe control cells (right column). Scales are different for each day. The intensity of the clusters at each time point, as quantified with Living Image (Perkin Elmer), is plotted in figure S10. 


\section{REFERENCES}

(1) Khlebtsov, B. N.; Khlebtsov, N. G. On the Measurement of Gold Nanoparticle Sizes by the Dynamic Light Scattering Method. Colloid J. 2011, 73, 118-127.

(2) Volkert, A. a.; Pierre, M. C. S.; Shrestha, B.; Haes, A. J. Implications of Sample Aging on the Formation of Internally Etched Silica Coated Gold Nanoparticles. RSC Adv. 2015, 5, 37743780.

(3) Nieves, D. J.; Li, Y.; Fernig, D. G.; Lévy, R. Photothermal Raster Image Correlation Spectroscopy of Gold Nanoparticles in Solution and on Live Cells. R. Soc. Open Sci. 2015, 2, 140454.

(4) Nečas, D.; Klapetek, P. Gwyddion: An Open-Source Software for SPM Data Analysis. Open Phys. 2012, 10, 181-188. 